\title{
Quantum resources for energy storage
}

\author{
Dario Ferraro ${ }^{1, *}$, Michele Campisi ${ }^{2,3}$, Gian Marcello Andolina ${ }^{4,5}$, Vittorio Pellegrini ${ }^{4,6}$, and Marco Polini ${ }^{4}$ \\ ${ }^{1}$ Dipartimento di Fisica, Universitá di Genova, Via Dodecaneso 33, I-16146 Genova, Italy \\ ${ }^{2}$ Dipartimento di Fisica e Astronomia, Universitá di Firenze, Via Sansone 1, I-50019 Sesto Fiorentino (FI), Italy \\ ${ }^{3}$ INFN Sezione di Firenze, Via G. Sansone 1, I-50019 Sesto Fiorentino (FI), Italy \\ ${ }^{4}$ Istituto Italiano di Tecnologia, Graphene Labs, Via Morego 30, I-16163 Genova, Italy \\ ${ }^{5}$ NEST, Scuola Normale Superiore, I-56126 Pisa, Italy \\ ${ }^{6}$ Bedimensional S.p.a., Via Albisola 121, I-16163 Genova, Italy
}

\begin{abstract}
Recently the possibility to exploit quantum-mechanical effects to increase the performance of energy storage has raised a great interest. It consists of $N$ two-level systems coupled to a single photonic mode in a cavity. We demonstrate the emergence of a quantum advantage in the charging power on this collective model (Dicke Quantum Battery) with respect to the one in which each two-level system is coupled to its own separate cavity mode (Rabi Quantum Battery). Moreover, we discuss the model of a Quantum Supercapacitor. This consists of two chains, one containing electrons and the other one holes, hosted by arrays of double quantum dots. The two chains are in close proximity and embedded in the same photonic cavity, in the same spirit of the Dicke model. We find the phase diagram of this model showing that, when transitioning from the ferro/antiferromagnetic to the superradiant phase, the quantum capacitance of the model is greatly enhanced.
\end{abstract}

\section{Introduction}

An ever increasing demand of high performance devices for energy storage represents one of the main issues for nowadays technological development [1]. In this context, batteries [2] and supercapacitors [3, 4] have found an extremely wide range of applications. These systems still operate on the basis of physical and chemical principles discovered between the Eighteenth and Nineteenth centuries [2]. However, new strategies are needed in order to overcome their intrinsic limitations. Although a great effort is currently focused on the material optimization [5], fundamental research in this field calls, in the long run, to a radical paradigmatic shift.

A very interesting possibility to enhance the average charging power of a battery or the energy stored in a supercapacitor is offered by quantum mechanics. Indeed, quantum phenomena are predicted to increase the performance of technological devices [6] and an increasing theoretical and experimental research activity is currently focused on applying quantum resources to improve energy storage and transfer [7-14] in the framework of the so called quantum thermodynamics. More specifically, a great number of researchers have recently addressed various aspects of quantum batteries [15-29]. A solid-state implementation of a quantum battery based on an array of $N$ two-level systems (TLSs) coupled to a common cavity photonic mode (known as Dicke model [30, 31]) has been introduced in Ref. [32]. Here, a $\sqrt{N}$ enhancement of the averaged charg-

*e-mail: ferraro@fisica.unige.it ing power, keeping substantially fixed the average stored energy, has been theoretically demonstrated.

Exploiting further the interesting physics related to light-matter interaction, in Ref. [33] a model for a Quantum Supercapacitor based on two chains of double quantum dots (DQDs) [34], one filled with electrons the other with holes, which implements a collections of TLSs has been introduced. All DQDs are coupled to a common photonic cavity mode [35-39]. Each chain separately can be seen as a realization of the Dicke-Ising model [40]. The two chains, however, are further coupled via an onsite electron-hole attractive interaction, which brings to an even richer phase diagram investigated by means of an analytical variational approach. As a remarkable consequence of the charge rearrangement that occurs at the superradiant phase transition [41] the capacitance of the system is strongly enhanced.

Aim of this paper is to review the physical mechanisms underlying both the Dicke quantum battery and the Quantum Supercapacitors realized by means of a Dicke-Ising interaction focussing also on their possible solid states implementations.

The present article is organized as follows. In Section 2 we discuss our model for the Dicke quantum battery showing the advantage associated to a collective charging. The physics of the quantum supercapacitor is described in Section 3. Experimentally feasible realizations of these devices are considered in Section 3.5. Finally Section 4 is devoted to Conclusions. 


\section{Dicke quantum battery}

\subsection{Model}

We consider a collection of $N$ TLSs, each being initially prepared in its ground state $|\mathrm{g}\rangle$. They are promoted to the excite state $|\mathrm{e}\rangle$ via coupling to a single cavity mode (charging process). Even if more general initial states can be considered [21], we assume for sake of simplicity that the cavity is in the $N$ photons Fock state $|N\rangle$. According to this, the initial state of the total system is:

$$
\left|\psi^{(N)}(0)\right\rangle=|N\rangle \otimes \underbrace{|\mathrm{g}, \mathrm{g}, \ldots, \mathrm{g}\rangle}_{N} .
$$

The quantum dynamics of this system is governed by the Dicke Hamiltonian [30]:

$$
\hat{\mathcal{H}}_{\lambda_{t}}^{(N)}=\hbar \omega_{\mathrm{c}} \hat{a}^{\dagger} \hat{a}+\omega_{\mathrm{a}} \hat{J}_{z}+2 \omega_{\mathrm{c}} \lambda_{t} \hat{J}_{x}\left(\hat{a}^{\dagger}+\hat{a}\right) .
$$

Here, $\hat{a}\left(\hat{a}^{\dagger}\right)$ annihilates (creates) a cavity photon with frequency $\omega_{\mathrm{c}}$ and

$$
\hat{J}_{\alpha}=\frac{\hbar}{2} \sum_{i}^{N} \hat{\sigma}_{i}^{\alpha} \quad \alpha=x, y, z
$$

are the components of a pseudospin operator expressed in terms of the Pauli operators $\hat{\sigma}_{i}^{\alpha}$ associated to the $i$-th TLS.

With $\hbar \omega_{\mathrm{a}}$ we indicate the level spacing between the ground and excited state of each TLS. Throughout all this paper, we will focus on the resonant regime, $\omega_{\mathrm{a}}=\omega_{\mathrm{c}}$. The dimensionless light-matter coupling $\lambda_{t}$ is driven in time according to the charging (discharging) protocol sketched in Figure 1.

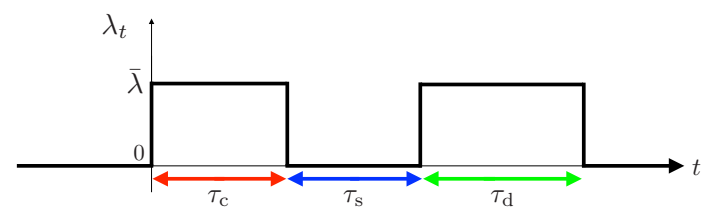

Figure 1. Evolution of the coupling $\lambda_{t}$ between the TLSs and the cavity radiation as a function of time. One can identify three different regions. Charging. Here, interaction is suddenly turned on at time $t=0^{+}$up to a constant value $\lambda_{0^{+}}=\bar{\lambda}$, and kept it at this value for $0<t \leq \tau_{\mathrm{c}}$. An energy transfer occurs from the cavity to the array of TLSs. Storage. The interaction is then turned off at time $\tau_{\mathrm{c}}^{+}$, i.e. $\lambda_{\tau_{\mathrm{c}}^{+}}=0$, and kept it off for $\tau_{\mathrm{c}}<t \leq \tau_{\mathrm{c}}+\tau_{\mathrm{s}}$. Here, the TLSs are assumed to be isolated from the environment, and hence keep their energy (as long as relaxation effects are negligible). Discharging. The interaction is again turned on for a time $\tau_{\mathrm{d}}$ with $\lambda_{t}=\bar{\lambda}$ for $\tau_{\mathrm{c}}+\tau_{\mathrm{s}}<t \leq \tau_{\mathrm{c}}+\tau_{\mathrm{s}}+\tau_{\mathrm{d}}$. In this phase energy is transferred back to the cavity. Picture taken from Ref. [32].

\subsection{Parallel charging}

Let's start by considering the case in which each of the $N$ TLSs is coupled to a separate cavity (parallel charging) as shown in Figure 2. Here, the Dicke Hamiltonian

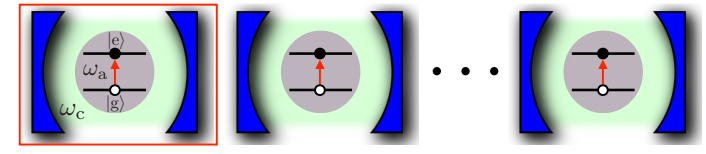

Figure 2. Array of identical TLSs charged in parallel (Rabi QB). The elementary unit (red box) consists of a two-level system with an energy separation $\hbar \omega_{\mathrm{a}}$ between the ground $|\mathrm{g}\rangle$ and excited state $|\mathrm{e}\rangle$. Each two-level system is coupled to a separate cavity (blue) hosting a single photonic mode. The red arrow indicates a transition induced by the radiation. Picture taken from Ref. [32].

in Eq. (2) reduces to $N$ identical copies of the the Rabi Hamiltonian [42, 43].

The energy stored in the system after at time $\tau_{\mathrm{c}}$ operating in this parallel fashion, indicated with $E_{\bar{\lambda}}^{(\|)}\left(\tau_{\mathrm{c}}\right)$, is $N$ times the energy $\epsilon_{\bar{\lambda}}\left(\tau_{\mathrm{c}}\right)$ stored in a single Rabi QB:

$$
\begin{aligned}
E_{\bar{\lambda}}^{(\|)}\left(\tau_{\mathrm{c}}\right)=N \epsilon_{\bar{\lambda}}\left(\tau_{\mathrm{c}}\right) \equiv \frac{N \hbar \omega_{\mathrm{c}}}{2} & {\left[\left\langle\psi_{\bar{\lambda}}^{(1)}\left(\tau_{\mathrm{c}}\right)\left|\hat{\sigma}_{z}\right| \psi_{\bar{\lambda}}^{(1)}\left(\tau_{\mathrm{c}}\right)\right.\right.} \\
& \left.-\left\langle\psi^{(1)}(0)\left|\hat{\sigma}_{z}\right| \psi^{(1)}(0)\right\rangle\right] .
\end{aligned}
$$

The label $\bar{\lambda}$ in $E_{\bar{\lambda}}^{(\mathrm{II})}\left(\tau_{\mathrm{c}}\right)$ reminds the fact that the stored energy depends on the coupling $\bar{\lambda}$ of the charging step. Moreover, one has

$$
\left|\psi_{\bar{\lambda}}^{(1)}\left(\tau_{\mathrm{c}}\right)\right\rangle=e^{-i \hat{\mathcal{H}}_{\bar{\lambda}}^{(1)} \tau_{\mathrm{c}} / \hbar}\left|\psi^{(1)}(0)\right\rangle .
$$

We now introduce the maximum stored energy (i.e. the “capacity' [24]')

$$
E_{\bar{\lambda}}^{(\|)}=\max _{\tau_{\mathrm{c}}}\left[E_{\bar{\lambda}}^{(\|)}\left(\tau_{\mathrm{c}}\right)\right]
$$

and the maximum charging power

$$
P_{\bar{\lambda}}^{(\|)}=\max _{\tau_{\mathrm{c}}}\left[P_{\bar{\lambda}}^{(\|)}\left(\tau_{\mathrm{c}}\right)\right] \equiv \max _{\tau_{\mathrm{c}}}\left[\frac{E_{\bar{\lambda}}^{(\mathbb{\|})}\left(\tau_{\mathrm{c}}\right)}{\tau_{\mathrm{c}}}\right] .
$$

According to Eq. (4) one has that both $E_{\bar{\lambda}}^{(\mathrm{Il})}$ and $P_{\bar{\lambda}}^{(\mathrm{ll})}$ scale linearly with $N$ (constant energy and power per QB).

\subsection{Collective charging}

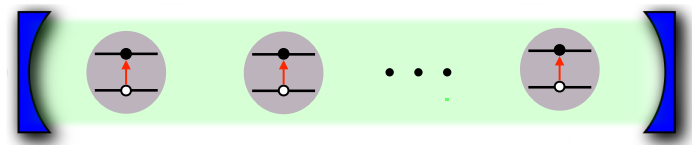

Figure 3. Array of identical TLSs charged in a collective way (Dicke QB), namely coupled to the same cavity. The red arrow indicates a transition induced by the radiation. Picture taken from Ref. [32].

We now investigate the capacity when the $N$ TLSs are coupled to a unique cavity (see Figure 3). It is described by the Dicke Hamiltonian in Eq. (2). Due to the fact that $\hat{J}^{2}=\sum_{\alpha=x, y, z} \hat{J}_{\alpha}^{2}$ is a conserved quantity $[44,45]$ a convenient basis is $|n, j, m\rangle$, where $n$ indicates the number of 
photons, $j(j+1)$ is the eigenvalue of $\hat{J}^{2}$, and $m$ denotes the eigenvalue of $\hat{J}_{z}$.

It is worth mentioning that the Dicke Hamiltonian doesn't conserve the number of photons. Therefore, in order to solve the system through exact diagonalization we need to truncate it by introducing a cutoff $N_{\mathrm{ph}}>N$ in such a way that a larger value of it, $N_{\mathrm{ph}}^{\prime}>N_{\mathrm{ph}}$, would not produce any noticeable difference in the results.

The energy $E_{\bar{\lambda}}^{(\sharp)}\left(\tau_{\mathrm{c}}\right)$ stored collectively after a time $\tau_{\mathrm{c}}$ by the $N$ TLSs is given by

$$
\begin{aligned}
E_{\bar{\lambda}}^{(\sharp)}\left(\tau_{\mathrm{c}}\right)=\omega_{\mathrm{c}}[ & \left\langle\psi_{\bar{\lambda}}^{(N)}\left(\tau_{\mathrm{c}}\right)\left|\hat{J}_{z}\right| \psi_{\bar{\lambda}}^{(N)}\left(\tau_{\mathrm{c}}\right)\right\rangle \\
& \left.-\left\langle\psi^{(N)}(0)\left|\hat{J}_{z}\right| \psi^{(N)}(0)\right\rangle\right],
\end{aligned}
$$

where $\left|\psi_{\bar{\lambda}}^{(N)}\left(\tau_{\mathrm{c}}\right)\right\rangle=e^{-i \hat{\mathcal{H}}_{\bar{\lambda}}^{(N)} \tau_{\mathrm{c}} / \hbar}\left|\psi^{(N)}(0)\right\rangle$
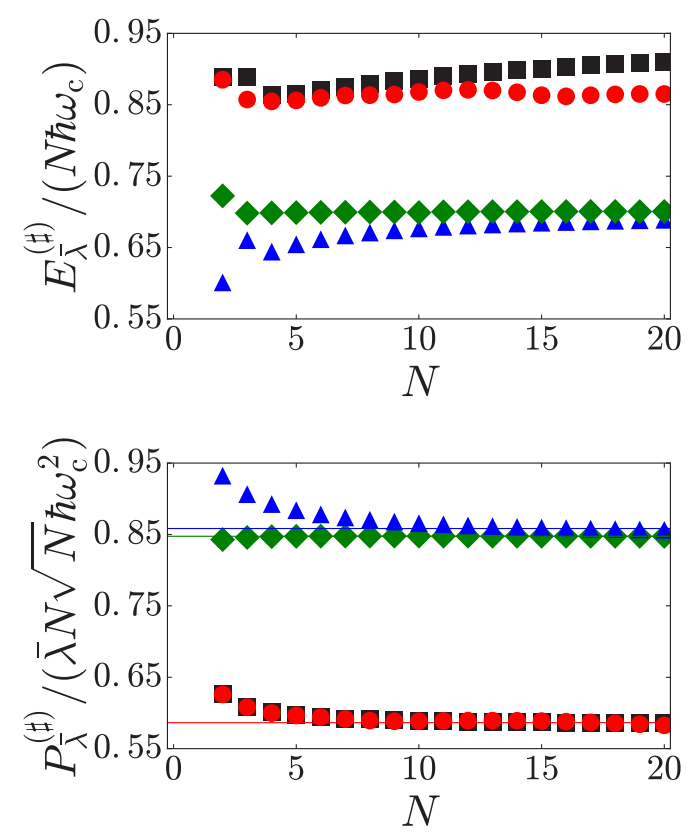

Figure 4. Top panel. Maximum collectively stored energy $E_{\bar{\lambda}}^{(\sharp)}$ (in units of $N \hbar \omega_{\mathrm{c}}$ ) as a function of $N$. Bottom panel. Maximum collective average charging power $P_{\bar{\lambda}}^{(\sharp)}$ (in units of $\bar{\lambda} N \sqrt{N} \hbar \omega_{\mathrm{c}}^{2}$ ) as a function of $N$. Black squares denote the results at $\bar{\lambda}=0.05$ neglecting the counter-rotating terms in Eq. (2). Results for Dicke QBs refer to $\bar{\lambda}=0.05$ (red circles), $\bar{\lambda}=0.5$ (blue triangles), and $\bar{\lambda}=2.0$ (green diamonds). The thin horizontal lines are best fits to the numerical results, indicating the asymptotic values of the maximum power at large $N$ : $\lim _{N \gg 1} P_{\bar{\lambda}}^{(\sharp)} /\left(\bar{\lambda} N \sqrt{N} \hbar \omega_{\mathrm{c}}^{2}\right)=0.586$ for $\bar{\lambda}=0.05$ (red), 0.858 for $\bar{\lambda}=0.5$ (blue), and 0.847 for $\bar{\lambda}=2$ (green).

Figures 4 show the maximum stored energy (top)

$$
E_{\bar{\lambda}}^{(\sharp)} \equiv \max _{\tau_{\mathrm{c}}}\left[E_{\bar{\lambda}}^{(\sharp)}\left(\tau_{\mathrm{c}}\right)\right]
$$

and maximum charging power (bottom)

$$
P_{\bar{\lambda}}^{(\sharp)} \equiv \max _{\tau_{\mathrm{c}}}\left[\frac{E_{\bar{\lambda}}^{(\sharp)}\left(\tau_{\mathrm{c}}\right)}{\tau_{\mathrm{c}}}\right]
$$

in the collective case (properly rescaled), as functions of $N$, for various values of $\bar{\lambda}$. We clearly see that such rescaled quantities rapidly converge to a certain asymptotic value as $N$ increases. This implies that, for sufficiently large values of $N, E_{\bar{\lambda}}^{(\sharp)}$ and $P_{\bar{\lambda}}^{(\sharp)}$ reach asymptotic values characterized by the following scaling laws

$$
E_{\bar{\lambda}}^{(\sharp)} \propto N
$$

and

$$
P_{\bar{\lambda}}^{(\sharp)} \propto N \sqrt{N} .
$$

The super-linear scaling of the maximum averaged charging power in Eq. (12) constitutes direct evidence of a $\sqrt{N}$ advantage associated to collective charging as compared to parallel charging. Such advantage is related to the scaling law of the time needed to reach the maximum power, namely $\bar{\tau}_{\mathrm{c}} \propto 1 / \sqrt{N}$, and has its origin in the renormalization of the effective light-matter coupling as a consequence of the collective interaction [46]. Thus our Dicke QBs realize in practice the powerful charging mechanism described in abstract terms in Refs. [17, 18].

\subsection{Storage and discharging}

In order for our system to properly work as a battery we need to assume that the storage time $\tau_{\mathrm{s}}$ is much shorter than any decoherence/relaxation time scale associated to the device. For what it concerns the discharging phases one has that in the parallel case, and in the weak coupling limit $\bar{\lambda} \ll 1$, the initial state in Eq. (1) can be recovered at the end of the discharging phase as far as the condition $\tau_{\mathrm{c}}+\tau_{\mathrm{d}}=\pi /\left(\bar{\lambda} \omega_{\mathrm{c}}\right)$ is fulfilled. In the collective case, as either $N$ or $\bar{\lambda}$ increases, such recoverability is lost. This is a signature of an interesting trade-off between averaged charging power and reversibility of the charging process. Highest values of the maximum power are achieved at strong coupling at the cost of a lower stored energy. Conversely, at weak coupling, one finds larger values of the maximum stored energy at the cost of lower values of the maximum power.

\section{Quantum supercapacitor}

\subsection{Model}

We are going to investigate now two arrays of double quantum dots (DQDs) [34], each having the voltage profile sketched in the top panel of Figure 5. Each DQD can be seen as a charge qubit that plays the role of the TLS discussed in the previous Section [47-50]. We consider two coupled chains, each one containing $M$ DQDs. The chemical potentials in the top (T) and the bottom (B) are tuned in such a way to host exactly one electron and one hole in each DQD respectively (see Figure 5). Such charge configuration has been chosen in order to mimic the two oppositely charged plates of a classical capacitor. The only two relevant electrostatic interaction contributions are indicated as $\mathcal{U}$ and $\mathcal{V}$ in Figure 5. Finally, in each DQD, the transitions between the energy levels are induced by absorption (emission) of photons from (into) the electromagnetic field of a cavity through a Dicke-link coupling extending what done in the previous Section. 


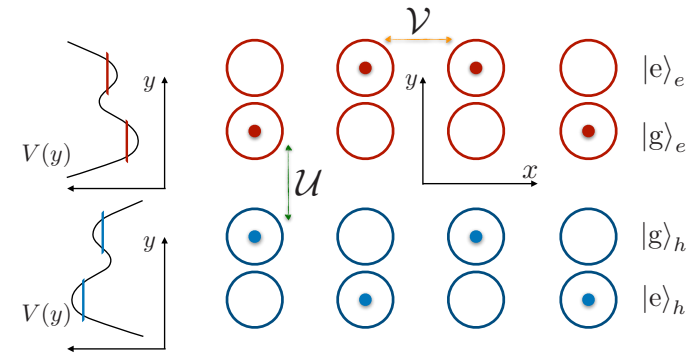

Figure 5. Schematic top view of the two-chain system. Here, one has a top (T) chain (red) and a bottom (B) chain (light blue) made up of DQDs. Each of them is singly occupied with electrons (dark red) and holes (dark blue) respectively thanks to the considered voltage profile $V(y)$. The two dominant contributions to the electrostatic interaction are: an inter-chain attractive interaction of strength $\mathcal{U}$ (green arrow) between an electron and a hole in their respective ground states; an intra-chain repulsive interaction of strength $\mathcal{V}$ (orange arrow) between electrons (holes) either in the ground-ground or in the excited-excited configuration. Picture taken from Ref. [33].

Accordingly, our model for a quantum supercapacitor is described by the Hamiltonian:

$$
\hat{\mathcal{H}}=\hat{\mathcal{H}}_{\mathrm{DI}}^{(\mathrm{T})}+\hat{\mathcal{H}}_{\mathrm{DI}}^{(\mathrm{B})}+\hat{\mathcal{H}}^{(\mathrm{TB})}+\hat{\mathcal{H}}^{(\mathrm{R})},
$$

where

$$
\begin{gathered}
\hat{\mathcal{H}}_{\mathrm{DI}}^{(\mathrm{T})}=\sum_{i=1}^{M}\left[\frac{\epsilon}{2} \hat{\tau}_{i}^{z}+\frac{\mathcal{V}}{2}\left(\hat{\tau}_{i}^{z} \hat{\tau}_{i+1}^{z}+1\right)+\hbar \omega_{\mathrm{c}} \lambda\left(\hat{a}^{\dagger}+\hat{a}\right) \hat{\tau}_{i}^{x}\right] \\
\hat{\mathcal{H}}^{(\mathrm{TB})}=-\frac{\mathcal{U}}{4} \sum_{i=1}^{M}\left(1-\hat{\tau}_{i}^{z}\right)\left(1-\hat{\sigma}_{i}^{z}\right)
\end{gathered}
$$

and

$$
\hat{\mathcal{H}}^{(\mathrm{R})}=\hbar \omega_{\mathrm{c}} \hat{a}^{\dagger} \hat{a} .
$$

The B chain Hamiltonian, $\hat{\mathcal{H}}_{\mathrm{DI}}^{(\mathrm{B})}$, can be obtained from $\hat{\mathcal{H}}_{\mathrm{DI}}^{(\mathrm{T})}$ by replacing $\hat{\tau}_{i}^{\alpha} \rightarrow \hat{\sigma}_{i}^{\alpha}$, where $\hat{\tau}_{i}^{\alpha}\left(\hat{\sigma}_{i}^{\alpha}\right)$ with $\alpha=x, z$ are pseudospin Pauli matrices acting on the 2D Hilbert space associated with the $i$-th DQD on the T (B) chain.

The above equation can be seen as two copies of the Dicke-Ising (DI) model introduced in Ref. [40], one for the $\mathrm{T}$ chain described by $\hat{\mathcal{H}}_{\mathrm{DI}}^{(\mathrm{T})}$ and one for the $\mathrm{B}$ chain described by $\hat{\mathcal{H}}_{\mathrm{DI}}^{(\mathrm{B})}$, further coupled by means of a local attractive interaction. The non-trivial phase diagram of the DI model in the $\mathcal{V}-\lambda$ space is inherited by our model. However, we expect additional ground-state phases due to the Coulomb attraction $\mathcal{U}>0$.

\subsection{Variational wave-function for the ground state and order parameters}

The ground state of the present system can be written in terms of the variational wave-function

$$
\begin{aligned}
|\Psi\rangle & =|\sqrt{M} \alpha\rangle \otimes \prod_{i=1}^{M}\left(\begin{array}{c}
\cos \left(\frac{\theta_{i}^{(\mathrm{B})}}{2}\right) \\
e^{i \chi_{i}^{(\mathrm{B})}} \sin \left(\frac{\theta_{i}^{(\mathrm{B})}}{2}\right)
\end{array}\right) \\
& \otimes \prod_{k=1}^{N}\left(\begin{array}{c}
\cos \left(\frac{\theta_{k}^{(\mathrm{T})}}{2}\right) \\
\left.e^{i \chi_{k}^{(\mathrm{T})} \sin \left(\frac{\theta_{k}^{(\mathrm{T})}}{2}\right)}\right)
\end{array}\right) .
\end{aligned}
$$

Here, $|\sqrt{M} \alpha\rangle$ is a photon coherent state in the cavity [41] and $\theta_{i}^{(\mathrm{T} / \mathrm{B})}, \chi_{i}^{(\mathrm{T} / \mathrm{B})}$ the angles characterizing the pseudospin associated with the $i$-th DQD of the T or B chain in the Bloch representation. In order to further simplify our analysis, we consider periodic boundary conditions $(M+1 \equiv 1)$ and we exploit the $\mathrm{T} \leftrightarrow \mathrm{B}$ exchange symmetry of the model, which allows us to set $\theta_{i}^{(\mathrm{T})}=\theta_{i}^{(\mathrm{B})}=\theta_{i}$ and $\chi_{i}^{(\mathrm{T})}=\chi_{i}^{(\mathrm{B})}=\chi_{i}$. The ground-state energy of the completely filled system is then given by

$$
\begin{aligned}
E & =\sum_{i=1}^{M}\left[\left(\epsilon+\frac{\mathcal{U}}{2}\right)\left(\cos \theta_{i}\right)+\mathcal{V}\left(\cos \theta_{i} \cos \theta_{i+1}\right)\right. \\
& -\frac{\mathcal{U}}{4}\left(\cos ^{2} \theta_{i}\right)+4 \hbar \omega_{\mathrm{c}} \lambda \sqrt{N} \alpha\left(\sin \theta_{i} \cos \chi_{i}\right) \\
& \left.+\hbar \omega_{\mathrm{c}} \alpha^{2}+\mathcal{V}-\frac{\mathcal{U}}{4}\right]
\end{aligned}
$$

Assuming $M$ to be even, and restricting the analysis to the case in which the polar $\theta_{i}$ and azimuthal $\chi_{i}$ angles can only change between even and odd sites [40], i.e. $\theta_{2 i+1}=\theta_{0}$, $\theta_{2 i}=\theta_{\mathrm{e}}, \chi_{2 i+1}=\chi_{\mathrm{o}}, \chi_{2 i}=\chi_{\mathrm{e}}$, we finally obtain

$$
\begin{aligned}
E & =M\left[\left(\frac{\epsilon}{2}+\frac{\mathcal{U}}{4}\right)\left(\cos \theta_{\mathrm{o}}+\cos \theta_{\mathrm{e}}\right)+\mathcal{V}\left(\cos \theta_{\mathrm{o}} \cos \theta_{\mathrm{e}}\right)\right. \\
& -\frac{\mathcal{U}}{8}\left(\cos ^{2} \theta_{\mathrm{o}}+\cos ^{2} \theta_{\mathrm{e}}\right) \\
& +2 \hbar \omega_{\mathrm{c}} \lambda \sqrt{N} \alpha\left(\sin \theta_{\mathrm{o}} \cos \chi_{\mathrm{o}}+\sin \theta_{\mathrm{e}} \cos \chi_{\mathrm{e}}\right) \\
& \left.+\hbar \omega_{\mathrm{c}} \alpha^{2}+\mathcal{V}-\frac{\mathcal{U}}{4}\right] .
\end{aligned}
$$

The function $E=E\left(\theta_{\mathrm{o}}, \theta_{\mathrm{e}}, \chi_{\mathrm{o}}, \chi_{\mathrm{e}}, \alpha\right)$ need to be minimized with respect to its variables in order to obtain the groundstate energy of the system and characterize the various possible phases. This leads to

$$
\begin{aligned}
\tilde{E} & =M\left[\left(\epsilon+\frac{\mathcal{U}}{2}\right) s+\mathcal{V}\left(s^{2}-m^{2}\right)\right. \\
& \left.-\frac{\mathcal{U}}{4}\left(s^{2}+m^{2}\right)-\hbar \omega_{\mathrm{c}} \mathcal{A}+\mathcal{V}-\frac{\mathcal{U}}{4}\right],
\end{aligned}
$$

where we have introduced the order parameters [40]

$$
\mathcal{A}=\frac{\left\langle\hat{a}^{\dagger} \hat{a}\right\rangle}{M}=\Lambda^{2}\left(\sin \theta_{\mathrm{o}}+\sin \theta_{\mathrm{e}}\right)^{2}
$$

which measures the average number of photons in the cavity and is non-zero in the superradiant phase;

$$
s=\frac{\left\langle\hat{\sigma}_{1}^{z}+\hat{\sigma}_{2}^{z}\right\rangle}{2}=\frac{1}{2}\left(\cos \theta_{\mathrm{o}}+\cos \theta_{\mathrm{e}}\right)
$$


is the magnetization of a plaquette composed of two neighboring sites and

$$
m=\frac{\left\langle\hat{\sigma}_{1}^{z}-\hat{\sigma}_{2}^{z}\right\rangle}{2}=\frac{1}{2}\left(\cos \theta_{\mathrm{o}}-\cos \theta_{\mathrm{e}}\right)
$$

which is the plaquette staggered magnetization.

\subsection{Phase diagram}

The quantity $\tilde{E}$ in Eq. (20) has been minimized numerically as a function of the dimensionless parameters $\tilde{\mathcal{U}} \equiv$ $\mathcal{U} / \hbar \omega_{\mathrm{c}}, \Lambda^{2} \equiv \lambda^{2} N$ and for different values of $\tilde{\mathcal{V}} \equiv$ $\mathcal{V} /\left(\hbar \omega_{\mathrm{c}}\right)$. One can identify four distinct phases (see Figure $6)$ :

- A ferromagnetic-normal (FN) phase ( $\mathcal{A}=0, s=-1$, and $m=0$ ), where electrons (holes) occupy the ground state $|\mathrm{g}\rangle_{e}\left(|\mathrm{~g}\rangle_{h}\right)$ of each DQD in the top (bottom) chain in absence of coherent cavity radiation.

- A ferromagnetic-superradiant (FS) phase ( $\mathcal{A} \neq 0, s \neq 0$, and $m=0$ ), where the radiation in the cavity becomes coherent.

- An antiferromagnetic-normal (AFN) phase $(\mathcal{A}=0, s=$ 0 , and $m=1$ ), where electrons occupy the ground state on even sites and the excited state on odd sites and holes in the bottom chain follow the same charge profile in absence of coherent cavity radiation.

- An antiferromagnetic-superradiant (AFS) phase ( $\mathcal{A} \neq 0$, $s \neq 0$, and $m \neq 0$ ), where the radiation in the cavity becomes coherent.

In top panel of Figure 6 we observe a net separation between the FN (blue) and FS phases (red), with a continuous transition occurring at

$$
\Lambda^{2}=\frac{1}{8}(1+\tilde{\mathcal{U}})
$$

By increasing $\tilde{\mathcal{V}}$ (bottom panels of Figure. 6) the FN phase progressively moves towards higher values of $\tilde{\mathcal{U}}$ and the AFN phase (green) emerges and expands, extending for small values of $\Lambda^{2}$ up to

$$
\tilde{\mathcal{U}}=4 \tilde{\mathcal{V}}-2,
$$

at which a first-order transition occurs. Moreover, at the boundary between FS and AFN phases a very narrow AFS region (yellow) appears.

It is worth noticing that the knowledge of all the three order parameters is needed to properly reconstruct the complete phase diagram.

\subsection{Quantum capacitance}

We can now consider the capacitance associated to our model. We can define it as the inverse of the discrete derivative of the chemical potential with respect to the number $M$ of charges [51, 52], i.e.

$$
C=\frac{e^{2}}{\mu_{M}-\mu_{M-1}},
$$
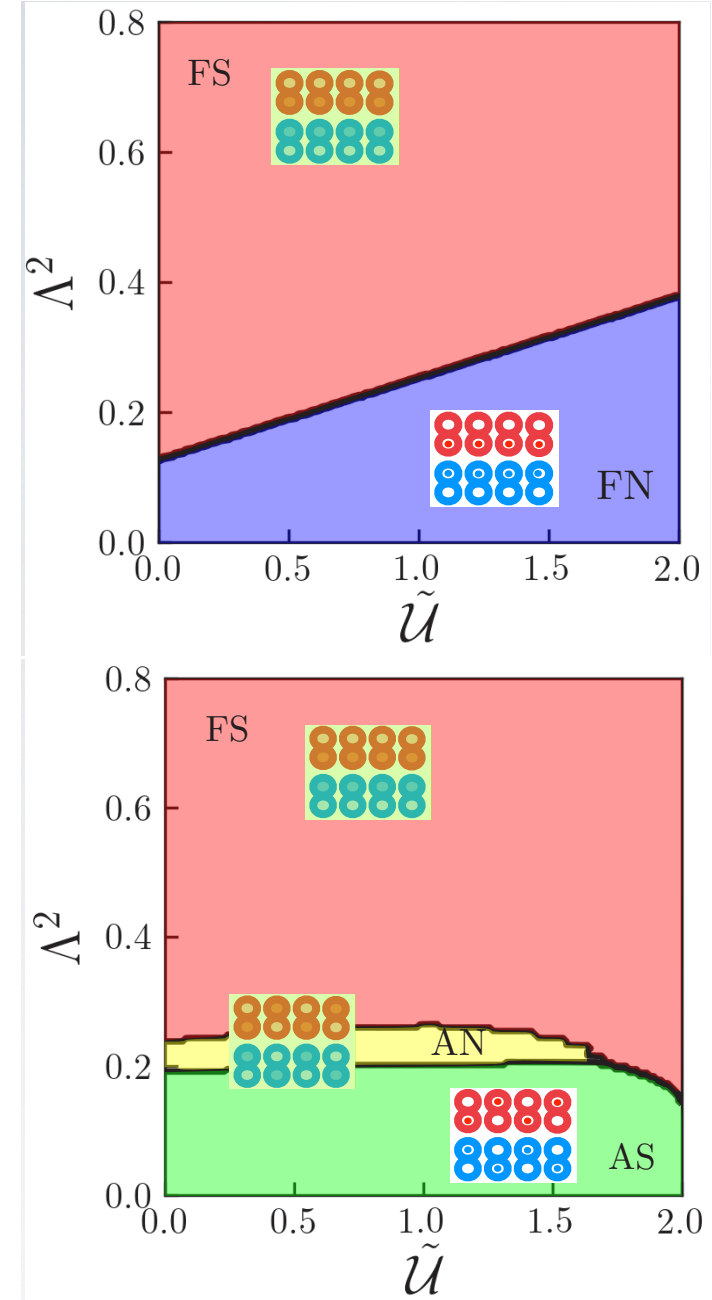

Figure 6. Phase diagram derived from Eq. (20) in the $\Lambda^{2}, \tilde{\mathcal{U}}$ space at different values of $\tilde{\mathcal{V}}$. Top panel. $\tilde{\mathcal{V}}=0$ case, the phase diagram shows a continuous phase transition between the ferromagnetic-normal (blue) and the ferromagnetic-superradiant (red) ordering. The situation remains qualitatively analogous up to $\tilde{\mathcal{V}} \lesssim 0.5$. Bottom panel. $\tilde{\mathcal{V}}=1.0$ case, emergence of both an antiferromagnetic-normal (green) and a narrow antiferromagnetic-superradiant (yellow) phase at the expense of the previously discussed ones. Insets represent the charge arrangement along the chain and the possible presence of radiation.

where $\mu_{M}=E_{M}-E_{M-1}$. Here, $E_{k}(k \in \mathbb{N})$ indicates the ground-state energy of the system where only $k$ sites of the chains (out of a total of $M$ sites per chain) are filled with electrons and holes. The problem is thereby reduced to evaluating the change in the ground-state energy of the system when an electron and a hole are removed from the same $i$-th site, while keeping fixed the total length of the two coupled chains. This protocol is motivated by the need to locally preserve the charge neutrality of the system, but explicitly breaks translational invariance.

Accordingly, we consider the following two-step protocol. One can first remove an electron-hole pair from the completely filled two-chain system in an arbitrary site (in the sublattice of odd sites to fix the notation). One has, in 
the $M \gg 1$ limit,

$$
\begin{aligned}
\mu_{M} & \approx \epsilon \cos \left(\theta_{\mathrm{o}}\right)-\frac{\mathcal{U}}{4}\left[1-\cos \left(\theta_{\mathrm{o}}\right)\right]^{2} \\
& +2 \mathcal{V}\left[\cos \left(\theta_{\mathrm{o}}\right) \cos \left(\theta_{\mathrm{e}}\right)+1\right]-8 \hbar \omega_{\mathrm{c}} \Lambda^{2} \sin ^{2}\left(\theta_{\mathrm{o}}\right) .
\end{aligned}
$$

A second electron-hole pair can be removed in one of the nearest-neighbor sites (both in the sublattice of even sites) leading to

$$
\begin{aligned}
\mu_{M-1} & \approx \epsilon \cos \left(\theta_{\mathrm{e}}\right)-\frac{\mathcal{U}}{4}\left[1-\cos \left(\theta_{\mathrm{e}}\right)\right]^{2} \\
& +\mathcal{V}\left[\cos \left(\theta_{\mathrm{o}}\right) \cos \left(\theta_{\mathrm{e}}\right)+1\right]-8 \hbar \omega_{\mathrm{c}} \Lambda^{2} \sin ^{2}\left(\theta_{\mathrm{e}}\right) .
\end{aligned}
$$

The above protocol reminds of what happens in atomic physics, where an atom with a completely filled shell is progressively ionized by removing the most loosely bound electrons and with $\mu_{M}\left(\mu_{M-1}\right)$ playing the role of first (second) ionization energy [51].

The capacitance of the system can then be written as

$$
C=\frac{C_{0}}{\left[(2+\tilde{\mathcal{U}}) m-\tilde{\mathcal{U}} m s+\tilde{\mathcal{V}}\left(1+s^{2}-m^{2}\right)+32 \Lambda^{2} m s\right]}
$$

with $C_{0} \equiv e^{2} / \hbar \omega_{\mathrm{c}}$ a dimensional factor.

In absence of coupling with the cavity radiation $\left(\Lambda^{2}=\right.$ 0), Eq. (29) reduces to

$$
C_{\mathrm{FN}}=\frac{C_{0}}{2 \tilde{V}}
$$

for the FN phase $(s=-1, m=0)$ and to

$$
C_{\mathrm{AFN}}=\frac{C_{0}}{(2+\tilde{\mathcal{U}})}
$$

(lower than $C_{\mathrm{FN}}$ in the considered range of parameters) for the AFN ( $s=0, m=1)$ phase. At finite values of the light-matter coupling, in the FS $(s \neq 0, m=0)$ phase, the capacitance becomes

$$
C_{\mathrm{FS}}=\frac{C_{0}}{\left(1+s^{2}\right) \tilde{\mathcal{V}}}
$$

In order to quantify the enhancement of the capacitance with respect to the one in absence of radiation, i.e. $C$ evaluated at $\Lambda^{2}=0$ and indicated with $\bar{C}$ in the following, we need to introduce the ratio

$$
\kappa=\frac{C}{\bar{C}}-1 .
$$

Its behavior as a function of the dimensionless parameters $\tilde{\mathcal{U}} \equiv \mathcal{U} / \hbar \omega_{\mathrm{c}}$ and $\Lambda^{2} \equiv \lambda^{2} N$ and for different values of $\tilde{\mathcal{V}} \equiv \mathcal{V} /\left(\hbar \omega_{\mathrm{c}}\right)$ is reported in Figure 7. It is positive and shows that an enhancement of the capacitance $(\kappa>0)$ associated to the superradiant phase transition occurs in the system due to the, purely non-classical, arrangement of the charges (see Figure 6). In particular, at the transition between the FN and FS phase (top panel of Figure 7), we have

$$
\kappa=\frac{C_{\mathrm{FS}}}{C_{\mathrm{FN}}}-1=\frac{1-s^{2}}{1+s^{2}} .
$$
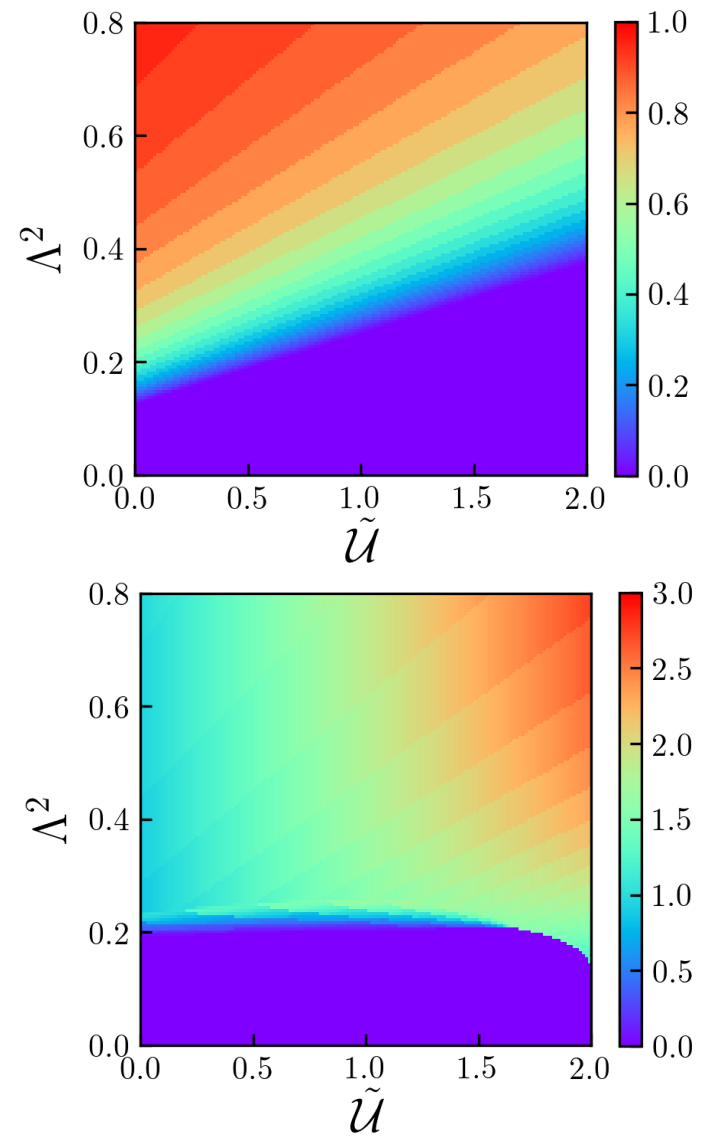

Figure 7. Density plots of the ratio $\kappa$ as a function of $\tilde{\mathcal{U}}$ and $\Lambda^{2}$ for $\tilde{\mathcal{V}}=0$ (top panel) and $\tilde{\mathcal{V}}=1.0$ (bottom panel). Picture taken from Ref. [33].

This quantity only depends on the pseudospin order parameter $s$ and reaches the value $\kappa=1$ (doubling of the capacitance) deeply in the FS phase (where one asymptotically approaches $s=0$, namely electrons and holes are completely delocalized in the DQDs due to the radiation). Differently, at the transition between the AFN and FS phase and neglecting the small AFS phase (bottom panel of Figure 7), we find

$$
\kappa=\frac{C_{\mathrm{FS}}}{C_{\mathrm{AFN}}}-1=\frac{2+\tilde{\mathcal{U}}}{\left(1+s^{2}\right) \tilde{\mathcal{V}}}-1,
$$

which depends on both the pseudospin order parameter $s$ and the specific values of the interaction terms leading to even large values of the ratio $(\kappa>2.5)$. Again, this quantity is maximal when $s=0$.

\subsection{Possible solid state implementations}

Systems made of engineered TLSs coupled with a cavity radiation have been realized with state-of-the-art solidstate technologies such as superconducting qubits coupled to line resonators $[46,53,54]$ or quantum dots combined with superconductive microwave circuits [38, 39, 55-57]. In such devices one has resonant frequencies $\omega_{\mathrm{c}} \approx \omega_{\mathrm{a}}$ in the $\mathrm{GHz}$ range and coupling $g_{0}=\bar{\lambda} \omega_{\mathrm{c}}$ around $10-100 \mathrm{MHz}$ leading to $\bar{\lambda} \approx 10^{-3}-10^{-2}$. However, recent experimental 
work has also demonstrated that the strong-coupling $\bar{\lambda} \approx 1$ can be reached [58-60]. Moreover, as stated above, the proposed charging/discharging protocol is meaningful as long as the relevant decoherence rate $\Gamma_{\phi}$ and the electron relaxation rate $\Gamma_{\mathrm{e}}$ satisfy $\Gamma_{\phi} \lesssim \Gamma_{\mathrm{e}}<g_{0}$, a condition which is typically fulfilled in experiments $[39,46,61]$. As a final remark, it is worth mentioning the fact that the actual possibility to explore the normal/superradiant phase transition in a real solid-state device has been debated at length due to the presence of an additional term $\propto\left(\hat{a}^{\dagger}+\hat{a}\right)^{2}$ (not considered here), which emerges from the minimal coupling between matter and cavity radiation [63-69]. However, according to recent calculations [70], superradiant phase transition is expected also in correlated materials embedded in photonic cavities.

For what it concerns more specifically the solid state implementations of our quantum supercapacitor model, capacitive couplings between DQDs up to $\mathcal{U} / h \approx 30 \mathrm{GHz}$ have been reported [62]. Accordingly, it can be possible, at least in principle, to explore a quite wide interval of values of $\tilde{\mathcal{U}}$. Moreover, one can also change this parameter by both acting on the distance between the two chains and changing the dielectric constant of the environment where the chains are embedded. An analogous discussion also holds for the intra-chain coupling $\mathcal{V}$ which can be charged acting on the distance between the DQDs along each chain.

\section{Conclusions}

We have reviewed some recent results concerning energy storage in quantum devices based on two-level systems coupled with a cavity radiation.

We have introduced the concept of a Dicke quantum battery where the interaction of an array of $N$ two-level systems with a common quantized electromagnetic mode creates entanglement. We observe a $\sqrt{N}$-fold enhancement of the scaling of the maximum charging power with respect to the parallel case (Rabi quantum battery), independent of the value of the light-matter interaction.

We have also investigated a model of a Quantum $\mathrm{Su}$ percapacitor made of two chains, one containing electrons and the other one holes, hosted by arrays of double quantum dots. Electron and hole feel screened Coulomb interactions and the whole system is embedded a photonic cavity, which is responsible for long-range coupling between all the qubits. We characterized the stable phases of this model by means of a variational approach showing that, when transitioning from the ferro/antiferromagnetic to the superradiant phase, the quantum capacitance of the model is strongly enhanced.

Our work offers the proper theoretical background for the experimental realization of a novel class of quantum devices where an improvement in the energy transfer and storage performance is achieved through purely quantum mechanical effects.

\section{References}

[1] G. Crabtree, Nature 526, S92 (2015).
[2] C. A. Vincent and B. Scrosati, Modern batteries. (Butterworth-Heinemann, Oxford, 1997).

[3] G. Wang, L. Zhang, and J. Zhang, Chem. Soc. Rev. 41, 797 (2012).

[4] S. P. S. Badwal, S. S. Giddey, C. Munnings, A. I. Bhatt, and A. F. Hollenkamp, Front. Chem. 2, 79 (2014).

[5] F. Bonaccorso, L. Colombo, G. Yu, M. Stoller, V. Tozzini, A. C. Ferrari, R. S. Ruoff, and V. Pellegrini, Science 347, 1246501 (2015).

[6] D. P. Di Vincenzo, Science 270, 255 (1995).

[7] M. Campisi, P. Hänggi, and P. Talkner, Rev. Mod. Phys. 83, 1653 (2011)

[8] M. Campisi and R. Fazio, Nat. Commun. 7, 11895 (2016).

[9] M. Horodecki and J. Oppenheim, Nat. Comm. 4, 2059 (2013).

[10] J. Goold, M. Huber, A. Riera, L. del Rio, and P. Skrzypczyk, J. Phys. A: Math. Theor. 49, 143001 (2016).

[11] S. Vinjanampathy and J. Anders, Contemp. Phys. 57, 545 (2016).

[12] P. Strasberg, G. Schaller, T. Brandes, and M. Esposito, Phys. Rev. X 7, 021003 (2016).

[13] B. Karimi and J. P. Pekola, Phys. Rev. B 94, 184503 (2016).

[14] B. Karimi, J. P. Pekola, M. Campisi, and R. Fazio, Quantum Sci. Technol. 2, 044007 (2017).

[15] R. Alicki and M. Fannes, Phys. Rev. E 87, 042123 (2013).

[16] K. V. Hovhannisyan, M. Perarnau-Llobet, M. Huber, and A. Acin, A., Phys. Rev. Lett. 111, 240201 (2013).

[17] F. C. Binder, S. Vinjanampathy, K. Modi, and J. Goold, New J. Phys. 17, 075015 (2015).

[18] F. Campaioli, F. A. Pollock, F. C. Binder, L. Céleri, J. Goold, S. Vinjanampathy, and K. Modi, Phys. Rev. Lett. 118, 150601 (2017).

[19] N. Friis and M. Huber, Quantum 2, 61 (2017).

[20] T. P. Le, J. Levinsen, K. Modi, M. Parish, and F. A. Pollock, Phys. Rev. A 97, 022106 (2018).

[21] G. M. Andolina, D. Farina, A. Mari, V. Pellegrini, V. Giovannetti, and M. Polini, M. Phys. Rev. B 98, 205423 (2018).

[22] G. M. Andolina, M. Keck, A. Mari, M. Campisi, V. Giovannetti, and M. Polini, Phys. Rev. Lett. 122, 047702 (2019).

[23] D. Farina, G. M. Andolina, A. Mari, M. Polini, and V. Giovannetti, Phys. Rev. B 99, 035421 (2019).

[24] S. Juliá-Farrè, T. Salamon, A. Riera, M. N. Bera, and M. Lewenstein, arXiv:1811.04005.

[25] Y.-Y. Zhang, T.-R. Yang, L. Fu, and X. Wang, Phys. Rev. E 99, 052106 (2019).

[26] G. M. Andolina, M. Keck, A. Mari, V. Giovannetti, and M. Polini, Phys. Rev. B 99, 205437 (2019).

[27] X. Zhang and M. Blaauboer, arXiv:1812.10139.

[28] F. Barra, Phys. Rev. Lett. 122, 210601 (2019). 
[29] F. Campaioli, F. A. Pollock, and S. Vinjanampathy, arXiv:1805.05507.

[30] R.H. Dicke, Phys. Rev. 93, 99 (1954).

[31] B. M. Garraway, Philos. Trans. Royal Soc. A 369, 1137 (2011).

[32] D. Ferraro, M. Campisi, G. M. Andolina, V. Pellegrini, and M. Polini, Phys. Rev. Lett. 120, 117702 (2018).

[33] D. Ferraro, G. M. Andolina, M. Campisi, V. Pellegrini, and M. Polini, Phys. Rev. B 100, 075433 (2019).

[34] W. G. van der Wiel, S. De Franceschi, J. M. Elzerman, T. Fujisawa, S. Tarucha, and L. P. Kouwenhoven, Rev. Mod. Phys. 75, 1 (2002).

[35] L. Childress, A. S. Sorensen, and M. D. Lukin, Phys. Rev. A 69, 042302 (2004).

[36] T. Frey, P. J. Leek, M. Beck, A. Blais, T. Ihn, K. Ensslin, and A. Wallraff, Phys. Rev. Lett. 108, 046807 (2012).

[37] H. Toida, T. Nakajima, and S. Komiyama, Phys. Rev. Lett. 110, 066802 (2013).

[38] J. Basset, D.-D. Jarausch, A. Stockklauser, T. Frey, C. Reichl, W. Wegscheider, T. M. Ihn, K. Ensslin, and A. Wallraff, Phys. Rev. B 88, 125312 (2013).

[39] A. Stockklauser, P. Scarlino, J. V. Koski, S. Gasparinetti, C. K. Andersen, C. Reichl, W. Wegscheider, T. Ihn, K. Ensslin, and A. Wallraff, Phys. Rev. X 7, 011030 (2017).

[40] Y. Zhang, L. Yu, J.-Q. Liang, G. Chen, S. Jia, and F. Nori, Sci. Rep. 4, 4083 (2014).

[41] C. Emary and T. Brandes, Phys. Rev. E 67, 066203 (2003).

[42] I.I. Rabi, Phys. Rev. 49, 324 (1936).

[43] I.I. Rabi, Phys. Rev. 51, 652 (1937).

[44] M. Tavis and F.W. Cummings, Phys. Rev. 170, 379 (1968).

[45] M. Tavis and F.W. Cummings, Phys. Rev. 188, 692 (1969).

[46] J. M. Fink, R. Bianchetti, M. Baur, M. Göppl, L. Steffen, S. Filipp, P. J. Leek, A. Blais, and A. Wallraff, Phys. Rev. Lett. 103, 083601 (2009).

[47] T. Hayashi, T. Fujisawa, H. D. Cheong, Y. H. Jeong, and Y. Hirayama, Phys. Rev. Lett. 91,226804 (2003).

[48] J. Gorman, D. G. Hasko, and D. A. Williams, Phys. Rev. Lett. 95, 090502 (2005).

[49] K. Wang, C. Payette, Y. Dovzhenko, P. W. Deelman, and J. R. Petta, Phys. Rev. Lett. 111, 046801 (2013).

[50] T. Ota, K. Hitachi, and K. Muraki, Sci. Rep. 8, 5491 (2018).
[51] G. J. Iafrate, K. Hess, J. B. Krieger, and M. Macucci, Phys. Rev. B 52, 10737 (1995).

[52] O. Prus, A. Auerbach, Y. Aloni, U. Sivan, and R. Berkovits, Phys. Rev. B 54, R14289 (1996).

[53] A. Wallraff, D.I. Schuster, A. Blais, L. Frunzio, R.S. Huang, J. Majer, S. Kumar, S.M. Girvin, and R.J. Schoelkopf, Nature 431, 162 (2004).

[54] J. Clarke and F.K. Wilhelm, Nature 453, 1031 (2008).

[55] B. Küng, C. Rössler, M. Beck, M. Marthaler, D. S. Golubev, Y. Utsumi, T. Ihn, and K. Ensslin, Phys. Rev. X 7, 011001 (2012).

[56] C. Rössler, D. Oehri, O. Zilberberg, G. Blatter, M. Karalic, J. Pijnenburg, A. Hofmann, T. Ihn, K. Ensslin, C. Reichl, and W. Wegscheider, Phys. Rev. Lett. 115, 166603 (2015).

[57] A. Hofmann, V.F. Maisi, C. Rössler, J. Basset, T. Krähenmann, P. Märki, T. Ihn, K. Ensslin, C. Reichl, and W. Wegscheider, Phys. Rev. B 93, 035425 (2016).

[58] F. Yoshihara, T. Fuse, S. Ashhab, K. Kakuyanagi, S. Saito, and K. Semba, Nature Phys. 13, 44 (2017).

[59] N. K. Langford, R. Sagastizabal, M. Kounalakis, C. Dickel, A. Bruno, F. Luthi, D. J. Thoen, A. Endo, and L. DiCarlo, Nat. Commun. 8, 1715 (2017).

[60] J. Braumüller, M. Marthaler, A. Schneider, A. Stehli, H. Rotzinger, M. Weides, and A. V. Ustinov, Nat. Commun. 8, 779 (2017).

[61] M. Hofheinz, E.M. Weig, M. Ansmann, R.C. Bialczak, E. Lucero, M. Neeley, A.D. O'Connell, H. Wang, J.M. Martinis, and A.N. Cleland, Nature 454, 310 (2008).

[62] D. R. Ward, D. Kim, D. E. Savage, M. G. Lagally, R. H. Foote, M. Friesen, S. M. Coppersmith, and M. A. Eriksson, npj Quantum Information 2, 16032 (2016).

[63] G. Chen, Z. Chen, and J. Liang, Phys. Rev. A 76, 055803 (2007).

[64] P. Nataf and C. Ciuti, Nat. Commun. 1, 72 (2010).

[65] O. Viehmann, J. von Delft, and F. Marquardt, Phys. Rev. Lett. 107, 113602 (2011).

[66] L. Chirolli, M. Polini, V. Giovannetti, and A. H. MacDonald, Phys. Rev. Lett. 109, 267404 (2012).

[67] F. M. D. Pellegrino, L. Chirolli, R. Fazio, V. Giovannetti, and M. Polini, Phys. Rev. B 89, 165406 (2014).

[68] F. M. D. Pellegrino, V. Giovannetti, A. H. MacDonald, and M. Polini, Nat. Commun. 7, 13355 (2016).

[69] A. F. Kockum, A. Miranowicz, S. De Liberato, S. Savasta, and F. Nori, Nat. Rev. Phys. 1, 19 (2019).

[70] G. Mazza and A. Georges, Phys. Rev. Lett. 122, 017401 (2019). 